\title{
What can magnetic early B-type stars tell us about early B-type stars in general?
}

\section{Matthew Shultz ${ }^{1}$, Gregg Wade ${ }^{2}$, Thomas Rivinius ${ }^{3}$, Coralie Neiner ${ }^{4}$, Evelyne Alecian ${ }^{5,6,7}$, Véronique Petit ${ }^{8}$, Jason Grunhut ${ }^{9}$ and the MiMeS \& BinaMIcS Collaborations}

${ }^{1}$ Department of Physics and Astronomy, Uppsala University, Box 516, Uppsala 75120

email: matthew.shultz@physics.uu.se

${ }^{2}$ Department of Physics, Royal Military College of Canada, Kingston, Ontario K7K 7B4, Canada

${ }^{3}$ ESO - European Organisation for Astronomical Research in the Southern Hemisphere, Casilla 19001, Santiago 19, Chile

${ }^{4}$ LESIA, Observatoire de Paris, PSL Research University, CNRS, Sorbonne Universités, UPMC Univ. Paris 06, Univ. Paris Diderot, Sorbonne Paris Cité, 5 place Jules Janssen, 92195 Meudon, France

${ }^{5}$ Université Grenoble Alpes, IPAG, F-38000 Grenoble, France ${ }^{6}$ CNRS, IPAG, F-38000 Grenoble, France

${ }^{7}$ LESIA, Observatoire de Paris, CNRS UMR 8109, UPMC, Université Paris Diderot, 5 place Jules Janssen, 92190, Meudon, France

${ }^{8}$ Department of Physics and Astronomy, University of Delaware, 217 Sharp Lab, Newark, DE 19716, USA

${ }^{9}$ Dunlap Institute for Astronomy and Astrophysics, University of Toronto, 50 St. George Street, Toronto, ON, M5S 3H4, Canada

\begin{abstract}
Some magnetic early B-type stars display $\mathrm{H} \alpha$ emission originating in their Centrifugal Magnetospheres (CMs). To determine the rotational and magnetic properties necessary for the onset of emission, we analyzed a large spectropolarimetric dataset for a sample of 51 B5-B0 magnetic stars. New rotational periods were found for 15 stars. We determined physical parameters, dipolar magnetic field strengths, magnetospheric parameters, and magnetic braking timescales. $\mathrm{H} \alpha$-bright stars are more rapidly rotating, more strongly magnetized, and younger than the overall population. We use the high sensitivity of magnetic braking to the mass-loss rate to test the predictions of Vink et al. (2001) and Krtička (2014) by comparing ages $t$ to maximum spindown ages $t_{\mathrm{S}, \max }$. For stars with $M_{*}<10 M_{\odot}$ this comparison favours the Krtička recipe. For the most massive stars, both prescriptions yield $t \ll t_{\mathrm{S}, \max }$, a discrepancy which is difficult to explain via incorrect mass-loss rates alone.
\end{abstract}

Keywords. stars: magnetic fields, stars: mass loss, stars: rotation, stars: early-type

\section{Introduction, Observations, \& Methodology}

Magnetic $\mathrm{OB}$ stars often display $\mathrm{H} \alpha$ emission originating in their corotating magnetospheres, which arise from magnetic confinement of their ionized winds (Babel \& Montmerle 1997; ud-Doula \& Owocki 2002). Petit et al. (2013) showed that the emission properties of magnetic early B-type stars can be explained by the presence of a Centrifugal Magnetosphere (CM), in which magnetically enforced corotation provides centrifugal support against gravitational infall of the magnetically confined plasma, thus enabling accumulation of plasma to optically thick densities around stars with relatively weak winds (Townsend \& Owocki 2005). A CM is expected when the Alfvén radius $R_{\mathrm{A}}$, or the maximum extent of closed magnetic loops within the magnetosphere, is greater than the Kepler corotation radius $R_{\mathrm{K}}$, or the radius at which gravitational and centrifugal forces 


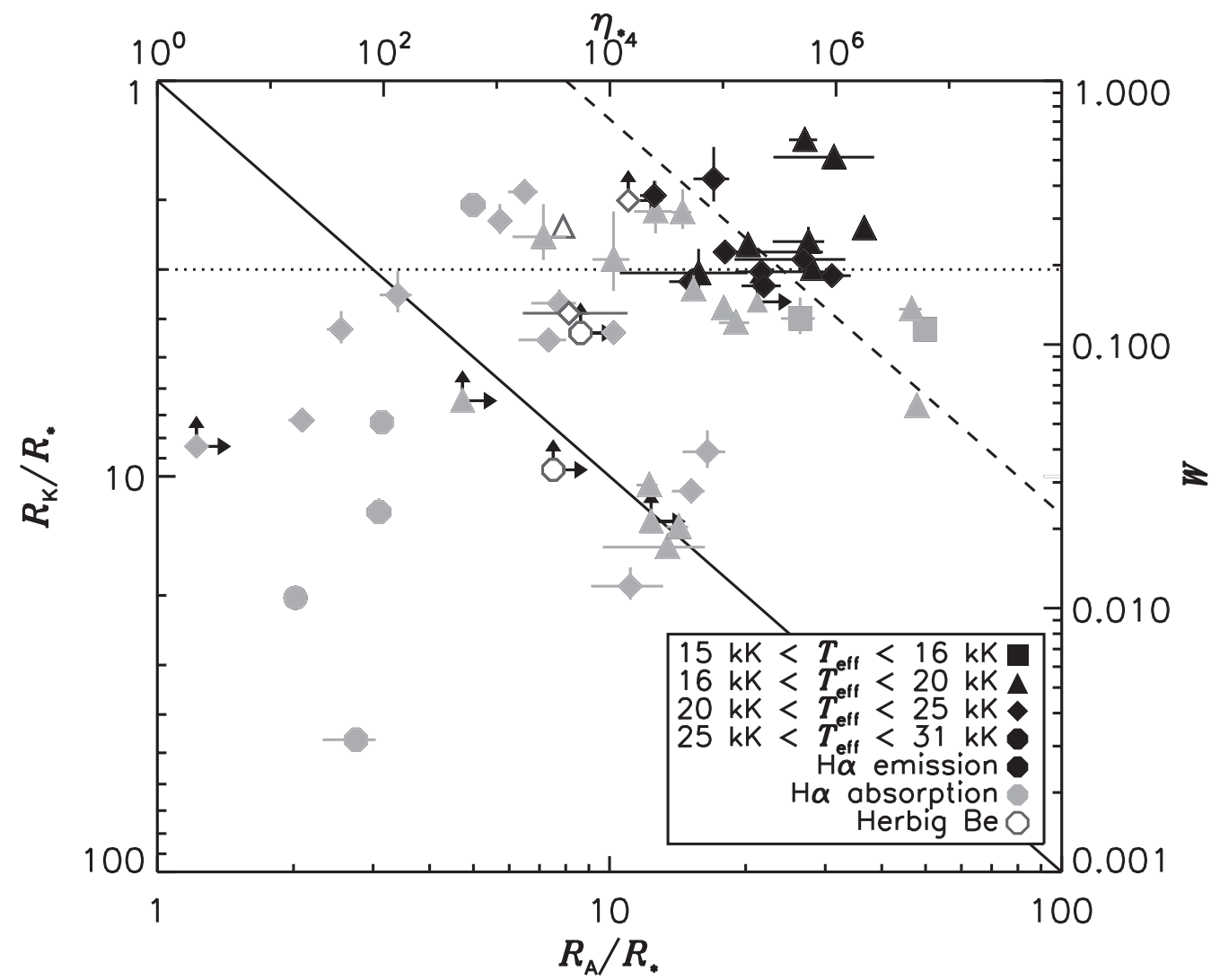

Figure 1. The rotation-magnetic confinement diagram, updated using newly determined $P_{\text {rot }}$ and $B_{\mathrm{d}}$. Alternate axes give the magnetic wind confinement parameter $\eta_{*}=\left(B_{\mathrm{d}}^{2} R_{*}^{2}\right) /\left(\dot{M} v_{\infty}\right)$, and the rotation parameter $W=v_{\text {rot }} / v_{\text {orb }}$ (where $v_{\text {orb }}$ is the velocity required for a Keplerian orbit at the stellar surface, and $W=1$ corresponds to the critical or breakup velocity). The diagnonal $R_{\mathrm{A}}=R_{\mathrm{K}}$ line divides stars with DMs (below) from those with CMs (above). The dotted line at $R_{\mathrm{K}}=2 R_{*}$ and the diagonal dashed line at $R_{\mathrm{A}}=8 R_{\mathrm{K}}$ divide stars with from stars without $\mathrm{H} \alpha$ emission.

are balanced. The absence of emission amongst many stars with $R_{\mathrm{A}}>R_{\mathrm{K}}$ indicates that merely possessing a CM is not a sufficient condition for the onset of $\mathrm{H} \alpha$ emission (Petit et al. 2013). However, owing to the relatively recent discovery of many of the known magnetic hot stars, their rotational periods and surface magnetic field strengths were not known, making it difficult to determine the conditions necessary for $\mathrm{H} \alpha$ emission.

To address this we assembled a sample consists of 51 main-sequence magnetic stars with spectral types between B5 and B0. The data (973 circularly polarized spectropolarimetric sequences) were obtained with ESPaDOnS at the $3.6 \mathrm{~m}$ Canada-France-Hawaii Telescope (CFHT), Narval at the $2 \mathrm{~m}$ Bernard Lyot Telescope, and HARPSpol at the European Southern Observatory (ESO) La Silla $3.6 \mathrm{~m}$ Telescope. The majority of the data were acquired by the Magnetism in Massive Stars (MiMeS) Large Programs (LPs). The instrumentation, data reduction, and the methodology utilized by the MiMeS LPs (in particular least-squares deconvolution of mean line profiles and measurement of the longitudinal magnetic field $\left.\left\langle B_{z}\right\rangle\right)$ are described by Wade et al. (2016). Some of the data were also acquired by the BinaMIcS LPs (Alecian et al. 2015) and the B-fields in OB stars (BOB) LP (Fossati et al. 2015), along with several CFHT and ESO PI programs. 

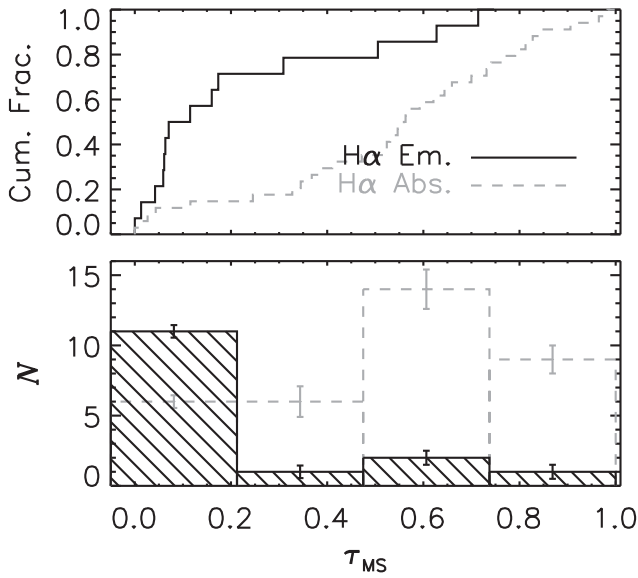

Figure 2. Cumulative distributions (top) and histograms (bottom) of $\tau_{\mathrm{MS}}$ for stars with and without $\mathrm{H} \alpha$ emission. Histogram errors were determined via Monte Carlo simulations using the formal uncertainties in $\tau_{\mathrm{MS}}$ obtained either from the HRD or cluster age MS turnoffs. $\mathrm{H} \alpha$-bright stars are heavily concentrated amongst the youngest stars, and indeed form a majority in this age-range $\left(\tau_{\mathrm{MS}}<0.25\right)$, despite making up only $29 \%$ of the overall population. The K-S test significance for the two distributions is $\sim 10^{-3}$, indicating they are distinct distributions with high probability.

Since many of the stars possess chemical abundance spots, photometric and spectroscopic as well as magnetic variability are all rotationally modulated. Rotational periods $P_{\text {rot }}$ were thus determined using Lomb-Scargle analysis of $\left\langle B_{z}\right\rangle$, as well as of archival photometric and/or spectroscopic time-series, when these data were available. $P_{\text {rot }}$ was determined for 15 of the 18 stars for which it was previously unknown.

Stellar parameters (radii $R_{*}$, masses $M_{*}$, ages $t$, and fractional main sequences ages $\left.\tau_{\mathrm{MS}}\right)$ were determined from the Hertzsprung-Russell diagram (HRD) and the $T_{\text {eff }}-\log g$ diagram, using the rotating evolutionary tracks calculated by Ekström et al. (2012). Cluster ages obtained from isochrone fitting to main-sequence turnoffs were used when available $\left(15 / 51\right.$ stars). Stellar parameters and $P_{\text {rot }}$ were used to obtain $R_{\mathrm{K}}$ (Townsend \& Owocki 2005). Surface magnetic field strengths $B_{\mathrm{d}}$ were determined from $\left\langle B_{z}\right\rangle$ and the stellar parameters using the method developed by Preston (1967). $R_{\mathrm{A}}$ and the maximum spindown age $t_{\mathrm{S}, \max }$ (ud-Doula \& Owocki 2002; ud-Doula et al. 2009) were calculated using the mass-loss rates $\dot{M}$ and wind terminal velocities $v_{\infty}$ obtained from the methods of both Vink et al. (2001) and Krtička (2014).

\section{Results}

Fig. 1 shows the rotation-magnetic confinement diagram introduced by Petit et al. (2013), updated with the newly determined magnetic and rotational parameters. $\mathrm{H} \alpha$ bright stars are concentrated in the upper right, in the region $R_{\mathrm{K}}<2 R_{*}$, and $R_{\mathrm{A}}>8 R_{\mathrm{K}}$. There are no stars in this region with $\mathrm{H} \alpha$ in absorption. This demonstrates that the rotational and magnetic properties of an early B-type star are reliable predictors of whether or not it will display $\mathrm{H} \alpha$ emission.

Since rapid rotation is a necessary condition for $\mathrm{H} \alpha$ emission, but the angular momentum of strongly magnetized stars is rapidly lost due to magnetic braking (ud-Doula et al. 2009), emission should be seen primarily in young stars. Fig. 2 demonstrates that this is in fact the case. The majority of $\mathrm{H} \alpha$-bright stars have $\tau_{\mathrm{MS}}<0.25$. Indeed, $\mathrm{H} \alpha$-bright stars are a majority $(11 / 17$ or $64 \%)$ of the stars in this age range, as compared to $15 / 51$ or $29 \%$ of the overall population.

Due to magnetic braking $P_{\text {rot }}$ should be longer for older stars. In Fig. 3a it can be seen that all stars with $P_{\text {rot }}>10 \mathrm{~d}$ are also older than $\tau_{\mathrm{MS}}>0.25$. Magnetic braking is highly sensitive to the mass-loss rate (ud-Doula et al. 2009), thus stars with higher masses, and stronger winds, should lose angular momentum more rapidly. As can be seen in Fig. 3a, the most slowly rotating stars in the sample are also amongst the most massive. 

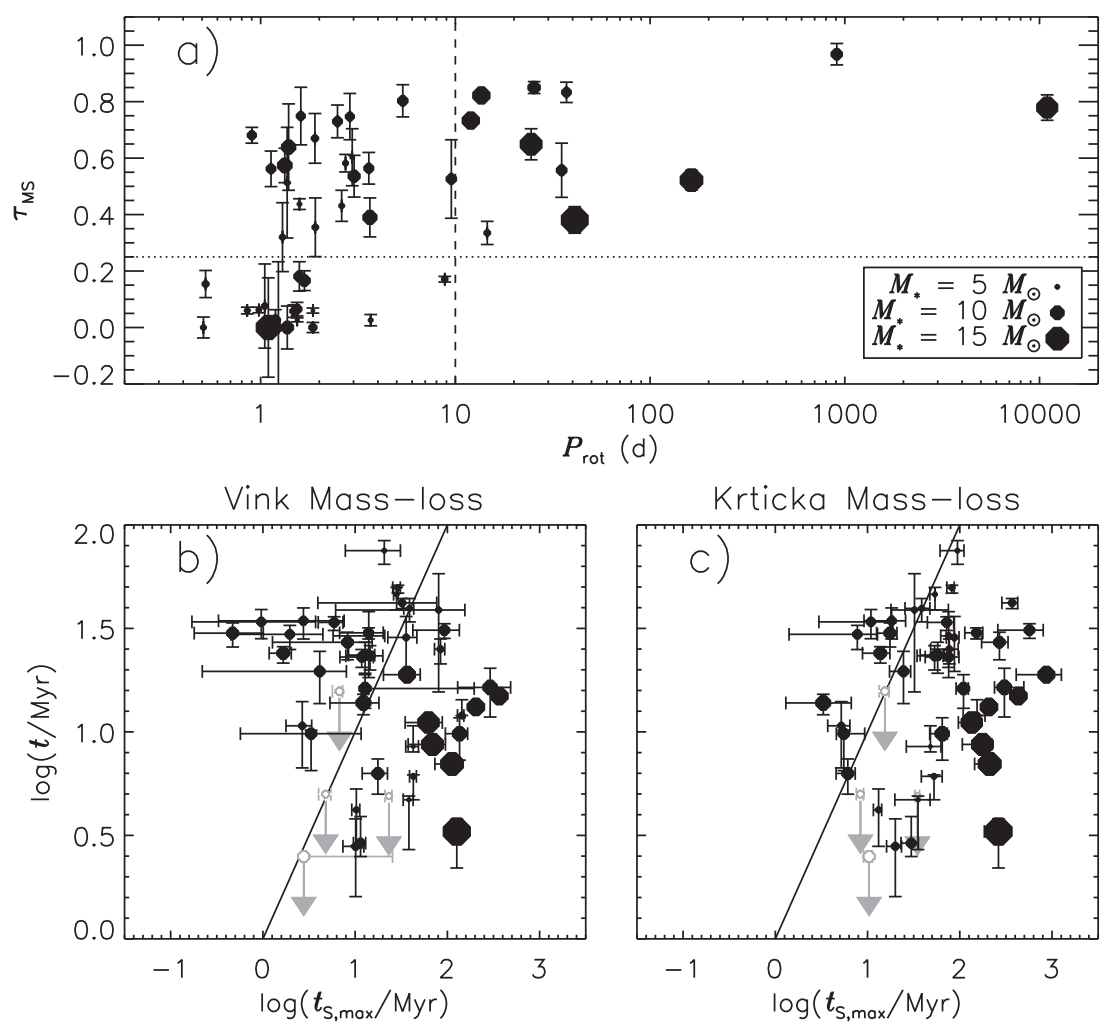

Figure 3. a) $\tau_{\mathrm{MS}}$ as a function of $P_{\mathrm{rot}}$. All stars with $P_{\mathrm{rot}}>10 \mathrm{~d}$ are older than $\tau_{\mathrm{MS}}=0.25$. More massive stars (which have stronger winds) tend to be more slowly rotating than less massive stars of similar age. $b$ ) the age $t$ inferred from the HRD, as a function of $t_{\mathrm{S}, \mathrm{max}}$ as computed with Vink mass-loss rates. Open symbols indicate stars with upper limits on $t$. The solid line shows $t=t_{\mathrm{S}, \max }$. There is poor agreement between the two timescales. $c$ ) as $b$, but computed with Krtička mass-loss rates. These yield a better agreement for the lower-mass stars, but cannot resolve the discrepancy for stars with $M_{*} \sim 15 M_{\odot}$, all of which are rotating much more slowly than predicted.

The bottom panels of Fig. 3 provide a more quantitative test of magnetic braking theory via direct comparison of the stellar age $t$ to the maximum spindown age $t_{\mathrm{S}, \max }$ (i.e. the time required for a star to spin down from critical rotation to its observed rotational velocity; ud-Doula et al. 2009). In Fig. $3 \mathrm{~b} t_{\mathrm{S}, \max }$ was calculated using Vink mass-loss, while in Fig. 3c the comparison is performed using Krtička mass-loss rates. Vink mass-loss results in $t_{\mathrm{S}, \max } \ll t$ for stars with $M_{*}<10 M_{\odot}$, with discrepancies up to 2 dex, i.e. the stars should be rotating much more slowly than observed. This is largely resolved by Krtička mass-loss rates. Both prescriptions yield similar solutions for highermass, hotter stars. For the most massive stars in the sample $\left(M_{*} \sim 15 M_{\odot}\right), t_{\mathrm{S}, \max } \gg t$; resolving this, again typically around $1 \mathrm{dex}$, would require $\dot{M}$ similar to values expected for O-type stars.

\section{Conclusions}

$\mathrm{H} \alpha$-bright CM-host stars are rapidly rotating, strongly magnetized, and young. Indeed, while $\mathrm{H} \alpha$ emission is fairly rare overall (occuring in about $1 / 4$ of the population), a majority (about 2/3) of young magnetic early B-type stars possess detectable CMs (Fig. 2). 
This suggests that young stellar clusters should be fertile ground for the detection of CMhost stars. The similar placement of all such stars on the rotation-magnetic confinement diagram (Fig. 1) suggests that the presence of CM emission is highly predictive of both rotational and magnetic properties. Future work will explore the sensitivity of emission strength to rotation and magnetic confinement strength.

The youth of CM-host stars, the increase in $P_{\text {rot }}$ over time, and the longer $P_{\text {rot }}$ typically observed for more massive stars, all serve as qualitative confirmation of rapid magnetic braking. The strong sensitivity of magnetic braking to mass-loss rates has enabled a test of competing models via comparison of $t$ to $t_{\mathrm{S}, \max }$. For stars with $M_{*}<10 M_{\odot}$, our results favour the mass-loss rates calculated by Krtička (2014). For more massive stars, neither Krtička nor Vink mass-loss rates are able to reconcile $t$ with $t_{\mathrm{S}, \max }$ (although Vink rates are marginally better in this mass range), and the discrepancy furthermore requires mass-loss rates similar to those of late O-type stars. As such high mass-loss rates are unlikely, alternative scenarios, e.g. rapid magnetic flux decay, strong magnetic braking on the pre-main sequence, or stellar structure modifications arising due to strong internal magnetic fields, will need to be explored.

\section{References}

Alecian E. et al., 2015, in IAU Symposium, Vol. 307, New Windows on Massive Stars, pp. $330-335$

Babel J., Montmerle T., 1997, ApJL, 485, L29

Ekström S. et al., 2012, $A \& A, 537$, A146

Fossati L. et al., 2015, $A \& A, 582$, A45

Krtička J., 2014, $A \& A, 564$, A70

Petit V. et al., 2013, MNRAS, 429, 398

Preston G. W., 1967, ApJ, 150, 547

Townsend R. H. D., Owocki S. P., 2005, MNRAS, 357, 251

ud-Doula A., Owocki S. P., 2002, ApJ, 576, 413

ud-Doula A., Owocki S. P., Townsend R. H. D., 2009, MNRAS, 392, 1022

Vink J. S., de Koter A., Lamers H. J. G. L. M., 2001, A\&A, 369, 574

Wade G. A. et al., 2016, MNRAS, 456, 2 